\title{
Prevalência de triatomíneos (Hemíptera: Reduviidae: Triatominae) infectados por Trypanosoma cruzi: sazonalidade e distribuição na região Ciénega do Estado de Jalisco, México
}

\author{
Prevalence of triatomines (Hemíptera: Reduviidae: Triatominae) infected \\ by Trypanosoma cruzi: seasonality and distribution in the \\ Ciénega region of the State of Jalisco, Mexico
}

\author{
César Gómez-Hernández ${ }^{1}$, Karine Rezende-Oliveira², Agustín Cortés Zárate ${ }^{3}$, \\ Esperanza Cortés Zárate ${ }^{3}$, Francisco Trujillo-Contreras ${ }^{4}$ e Luis Eduardo Ramirez ${ }^{1}$
}

\begin{abstract}
RESUMO
As características físico-geográficas da região Ciénega, Jalisco, México a tornam propícia para transmissão do Trypanosoma cruzi, causador da doença de Chagas. Este trabalho caracteriza a prevalência de triatomíneos infectados pelo parasita, sua sazonalidade e distribuição nesta região. Foram analisados 328 triatomíneos no período de janeiro de 2005 a junho de 2007 procedentes de 13 municípios da região, sendo abril, maio e junho os meses de maior captura. Dos triatomíneos analisados, 57,3\% foram positivos para Trypanosoma cruzi, correspondendo 15,4\% para a área urbana e 84,6\% para a área rural. A espécie mais freqüente foi Triatoma longipennis e a mais parasitada foi Triatoma barberi com índice de infecção de 83,3\% quando comparada a Triatoma longipennis $(67,5 \%)(\mathrm{p}<0,05)$. A infecção natural dos vetores capturados pode indicar alta exposição das pessoas ao Trypanosoma cruzi. 0 achado recente do Triatoma dimidiata positivo nesta região, sugere a adaptação de novas espécies às condições ecológicas destas populações.
\end{abstract}

Palavras-chaves: Trypanosoma cruzi. Triatomíneos. Doença de Chagas.

\section{ABSTRACT}

The physical and geographical characteristics of the Ciénega region, Jalisco, Mexico make it suitable for transmission of Trypanosoma cruzi, the causative agent for Chagas disease. This study characterizes the prevalence of triatomines infected by this parasite, their seasonality and their distribution in this region. A total of 328 triatomines were evaluated between January 2005 and June 2007, from 13 municipalities in the region. April, May and June were the months with the highest capture levels. Among the triatomines examined, 57.3\% were positive for Trypanosoma cruzi, corresponding to $15.4 \%$ in urban areas and 84.6\% in rural areas. The species with greatest prevalence was Triatoma longipennis and the species with the highest parasitism rate was Triatoma barberi, with an infection rate of $83.3 \%$, whereas the rate for Triatoma longipennis was $67.5 \%(\mathrm{p}<0.05)$. This natural infection in the captured vectors may indicate that individuals in this region have high exposure to Trypanosoma cruzi. The recent findings of positive Triatoma dimidiata in this region suggest that new species are becoming adapted to the ecological conditions of these populations.

Key-words: Trypanosoma cruzi. Triatomines. Chagas disease.

A doença de Chagas, também conhecida como tripanossomíase americana é considerada um problema de saúde pública em 17 países latino-americanos. Cerca de 10 a 12 milhões de pessoas estão infectadas com o Trypanosoma cruzi, sendo esta uma das principais doenças transmitidas por insetos triatomíneos, na América Latina e endêmica em vários estados do México ${ }^{724}$.
No México, existem 33 espécies de triatomíneos ${ }^{10}$ dos quais 18 têm sido encontradas infectadas naturalmente por Trypanosoma $c r u z i^{2226}$. No Estado de Jalisco, tem-se conhecimento destes insetos em decorrência de estudos realizados por Magallón-Gastélum e $\mathrm{col}^{16}$ e Martinez-Ibarra e col $^{17}$ mostrando sua distribuição estadual e sua relação com a infecção natural, considerando os complexos

\footnotetext{
1. Laboratório de Parasitologia, Universidade Federal do Triângulo Mineiro, Uberaba, MG. 2. Laboratório de Imunologia, Universidade Federal do Triângulo Mineiro, Uberaba, MG. 3. Secretaria de Salud Jalisco (SSJ), Región Sanitaria IV, Jalisco México. 4. Centro Universitario de los Altos (UdeG), Universidad de Guadalajara, Jalisco, México. Endereço para correspondência: Dr. Luis Eduardo Ramirez. Laboratório de Parasitologia/UFTM. Av. Getúlio Guaritá s/n, Bairro Abadia, $38025-440$ Uberaba, MG Tel: $55343318-5258$.

e-mail: parasito_fmtm@mednet.com.br

Recebido para publicação em: 21/11/2007

Aceito em: 09/05/2008
} 
Phylosoma e Proctata os mais importantes no processo de transmissão do Trypanosoma cruzi ${ }^{16}{ }^{17}$. A distribuição destes complexos está relacionada com índices de dispersão, infestação e infecção por Trypanosoma cruzi junto a fatores ecológicos e, sobretudo, aos hábitos domiciliares do inseto e à presença da doença de Chagas ${ }^{12} 1626$. Têm-se descrito em alguns municípios deste estado índices elevados de infestação que variam de 26,1\% a 58,3\%, colonização de triatomíneos no peridomicílio de 58,3\% a 85,7\% e infecção por Trypanosoma cruzi de 41,2\% a 60,2\%, os quais aumentam durante a temporada seca ${ }^{16}$.

Estudo realizado em 124 municípios do estado de Jalisco, entre 1987-1994 demonstrou uma soroprevalência da doença de Chagas na população geral de $18 \%$ em 124 municípios ${ }^{5}$. Recentemente, Yerenas e cols ${ }^{25}$, no ano de 2006, encontraram no município de La Barca, Jalisco, uma soroprevalência de 21,6\% na população rural ${ }^{25}$.

Devido ás elevadas taxas de prevalência de anticorpos na população de diferentes áreas do estado de Jalisco, aos elevados índices de infestação, colonização e infecção dos triatomíneos pelo Trypanosoma cruzi, nosso grupo de pesquisa realizou um estudo transversal, descritivo e observacional na região Ciénega, considerada montanhosa, localizada neste mesmo estado com objetivo de avaliar a prevalência de triatomíneos infectados pelo Trypanosoma cruzi, sua sazonalidade e distribuição nesta região.

\section{MATERIAL E MÉTODOS}

A região Ciénega apresenta uma superfície total de $4.892 \mathrm{~km}^{2}$ equivalente a $6,1 \%$ da superfície do Estado de Jalisco. A altitude da região é de $1.564 m$ ao nível do mar (manm), com alturas mínimas de 1.520 manm nos municípios de Poncitlán e La Barca e máximas de 1.780 manm no município de Degollado. A temperatura média regional é de $20,2^{\circ} \mathrm{C}$, com mínimas de $19,5^{\circ} \mathrm{C}$ em Tuxcueca e Jocotopec e máximas de $21,1^{\circ} \mathrm{C}$ em Atotonilco e Ayotlán, sendo seu clima semiquente a semi-seco. Esta região tem 463.039 habitantes, abrangendo um dos índices mais altos da população total do Estado de Jalisco ${ }^{20}$, representando uma densidade populacional de 94,65 habitantes/ $\mathrm{km}^{2}$ (Figura 1).

Foi realizada uma busca de triatomíneos peri e intradomiciliar durante o período de janeiro de 2005 a junho de 2007 em 13 municípios da região: Atotonilco, Ayotlán, Chapala, Degollado, Jamay, Jocotepec, La Barca, Ocotlán, Poncitlán, Tizapán, Tototlán, Tuxcueca, Zapotlán del Rey (Tabela 1).

A coleta foi manual com auxílio de pinças e lâmpadas, não sendo utilizadas armadilhas. 0 tempo de captura foi de aproximadamente uma hora/homem/casa ${ }^{15}$. Os triatomíneos coletados foram colocados em recipientes de plástico rotulados com endereço e data da captura e enviados para identificação e diagnóstico de Trypanosoma cruzi ao laboratório de Secretaria de Salúd Jalisco. A identificação dos triatomíneos foi realizada utilizando os critérios da classificação de Triatoma do México de Lent \& Wigodzinski1 ${ }^{14}$. A freqüência de captura nos municípios foi uma vez a cada 30 dias, e os endereços foram selecionados utilizando a tabela de números aleatórios. Ao todo, foram analisados 340 (10\%) domicílios.

A procura do Trypanosoma cruzi nas fezes dos triatomíneos foi realizada pelo exame á fresco pela compressão do abdômen e

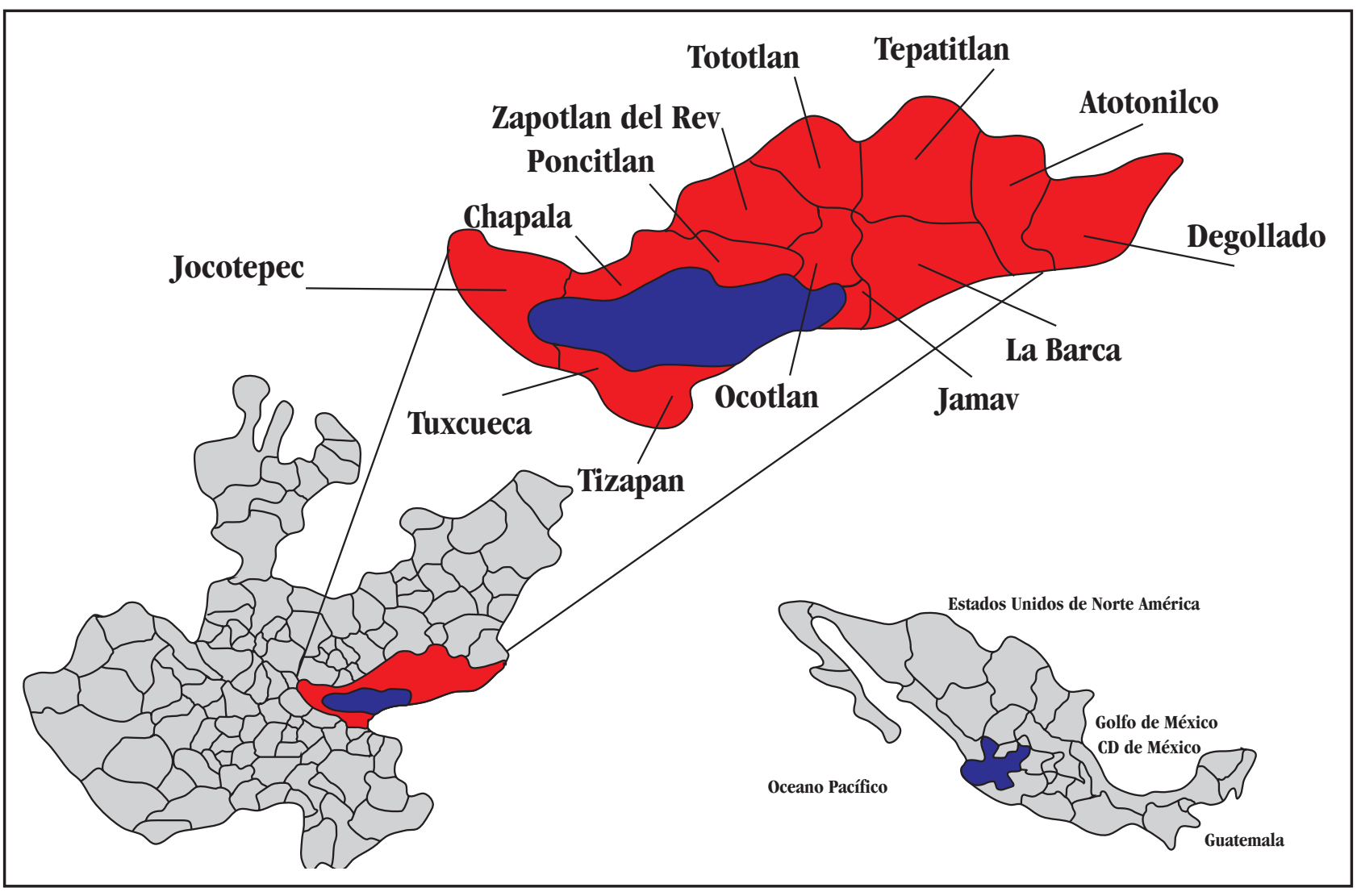

Figura 1 - Região Ciénega, Estado de Jalisco, México. 
Tabela 1 - Distribuição de triatomíneos por espécie e com infecção natural por Trypanosoma cruzi capturados em municípios da região Ciénega do Estado de Jalisco no período 2005 - 2007.

\begin{tabular}{|c|c|c|c|c|c|}
\hline \multirow[t]{2}{*}{ Município } & \multirow[t]{2}{*}{ Espécies } & \multicolumn{2}{|c|}{ Triatomíneos $\left(\mathrm{n}^{\circ}\right)$} & \multirow[t]{2}{*}{ IINPE } & \multirow[t]{2}{*}{ IIN } \\
\hline & & capturados & positivos & & \\
\hline \multirow[t]{3}{*}{ Atotonilco } & Triatoma longipennis & 14 & 8 & 57,1 & 69,5 \\
\hline & Triatoma barberi & 5 & 5 & 100,0 & \\
\hline & ninfas $s p$ & 4 & 3 & 75,0 & \\
\hline \multirow[t]{3}{*}{ Ayotlán } & Triatoma longipennis & 12 & 7 & 58,3 & 43,7 \\
\hline & Triatoma barberi & 0 & 0 & 0,0 & \\
\hline & ninfas $s p$ & 4 & 0 & 0,0 & \\
\hline \multirow[t]{3}{*}{ Chapala } & Triatoma longipennis & 6 & 4 & 66,6 & $\overline{60,0}$ \\
\hline & Triatoma barberi & 1 & 1 & 100,0 & \\
\hline & ninfas $s p$ & 3 & 1 & 33,3 & \\
\hline \multirow[t]{3}{*}{ Degollado } & Triatoma longipennis & 11 & 8 & 72,7 & 75,0 \\
\hline & Triatoma barberi & 1 & 1 & 100,0 & \\
\hline & $\operatorname{ninfas} s p$ & 0 & 0 & 0,0 & \\
\hline \multirow[t]{3}{*}{ Jamay } & Triatoma longipennis & 8 & 7 & 87,5 & $\overline{75,0}$ \\
\hline & Triatoma barberi & 5 & 5 & 100,0 & \\
\hline & ninfas $s p$ & 3 & 0 & 0,0 & \\
\hline \multirow[t]{3}{*}{ Jocotepec } & Triatoma longipennis & 25 & 21 & 84,0 & 67,6 \\
\hline & Triatoma barberi & 1 & 0 & 0,0 & \\
\hline & ninfas $s p$ & 8 & 3 & 37,5 & \\
\hline \multirow[t]{4}{*}{ La Barca } & Triatoma longipennis & 21 & 15 & 71,4 & 33,7 \\
\hline & Triatoma barberi & 15 & 11 & 73,3 & \\
\hline & Triatoma dimidiata & 1 & 1 & 100,0 & \\
\hline & $\operatorname{ninfas} s p$ & 46 & 1 & 2,1 & \\
\hline \multirow[t]{3}{*}{ Ocotlán } & Triatoma longipennis & 6 & 5 & 83,3 & $\overline{94,4}$ \\
\hline & Triatoma barberi & 12 & 12 & 100,0 & \\
\hline & $\operatorname{ninfas} s p$ & 0 & 0 & 0 & \\
\hline \multirow[t]{3}{*}{ Poncitlán } & Triatoma longipennis & 30 & 16 & 53,3 & 56,5 \\
\hline & Triatoma barberi & 3 & 2 & 66,6 & \\
\hline & ninfas $s p$ & 13 & 8 & 61,5 & \\
\hline \multirow[t]{3}{*}{ Tizapan } & Triatoma longipennis & 4 & 2 & 50,0 & 50,0 \\
\hline & Triatoma barberi & 2 & 1 & 50,0 & \\
\hline & ninfas $s p$ & 4 & 2 & 50,0 & \\
\hline \multirow[t]{3}{*}{ Tototlán } & Triatoma longipennis & 1 & 1 & 100,0 & 50,0 \\
\hline & Triatoma barberi & 1 & 0 & 0,0 & \\
\hline & ninfas $s p$ & 0 & 0 & 0,0 & \\
\hline \multirow[t]{3}{*}{ Tuxcueca } & Triatoma longipennis & 23 & 16 & 69,5 & \\
\hline & Triatoma barberi & 0 & 0 & 0,0 & \\
\hline & ninfas $s p$ & 9 & 4 & 44,4 & 62,5 \\
\hline \multirow[t]{3}{*}{ Zapotlán del Rey } & Triatoma longipennis & 20 & 14 & 70,0 & \\
\hline & Triatoma barberi & 2 & 2 & 100,0 & 69,2 \\
\hline & ninfas $s p$ & 4 & 2 & 50,0 & \\
\hline Total & & 328 & 188 & & 57.3 \\
\hline
\end{tabular}

IINPE: índice de infecção natural por espécie, IIN: índice de infecção natural.

pelo esfregaço corado pelo Giemsa para identificação morfológica dos parasitas ${ }^{16}$.

0 cálculo dos índices entomológicos de distribuição geográfica foi realizado de acordo com a definição da Organização Mundial de Saúde (WHO) ${ }^{24}$ onde se considera 0 índice de infecção natural (número de triatomíneos infectados x 100/número de triatomíneos capturados) e índice de infestação (número de unidades domiciliares com presença de triatomíneos x 100/número de unidades domiciliares pesquisadas).
A análise estatística foi realizada utilizando o método de quiquadrado de Fisher para comparação entre a prevalência das diferentes espécies de triatomíneos capturados $(\mathrm{p}<0,05)$. 0 s dados foram analisados com o auxílio dos programas Statistica, 6.0 e Statview for Windows.

\section{RESULTADOS}

Foram coletados no período de 2005-2007 um total de 328 exemplares em 13 municípios da região Cienega: Atotonilco (23), Ayotlán (16), Chapala (10), Degollado (12), Jamay (16), Jocotepec (34), La Barca (83), Ocotlán (18), Poncitlán (46), Tizapán (10), Tototlán (2), Tuxcueca (32), Zapotlán del Rey (26) (Tabela 1).

A porcentagem de exemplares coletados em 2005 foi de 38,4\%, em 2006 de 45,1\% e em 2007 de 16,4\%, sendo La Barca, Poncitlán, Jocotepec e Tuxcueca os que apresentaram o maior índice de captura 59,4\% (195/328). A prevalência de triatomíneos foi: Triatoma longipennis 182/328 (55,4\%) que apresentou um índice de infestação de 18,7\% na área intradomiciliar urbana, 26,9\% na área intradomiciliar rural e 53,8\% na região peridomiciliar rural. Triatoma barberi 50/328 (15,2\%), com índice de infestação de 3,3\% na área intradomiciliar urbana, 3,9\% na área intradomiciliar rural e 19,2\% na região peridomiciliar rural e foram capturadas $98(29,8 \%)$ ninfas $s p$, sendo 6\% encontradas na área intradomiciliar urbana, 6,6\% na área intradomiciliar rural e $41,2 \%$ na região peridomiciliar rural (Tabela 2 ). Os maiores índices de captura de triatomíneos foram observados nos meses de abril, maio e junho, correspondendo ao período de fim do inverno, época da seca e período anterior às chuvas (Figura 2 e Tabela 3).

Dos 328 triatomíneos capturados, 188 (57,3\%) apresentaram infecção natural por Trypanosoma cruzi, sendo encontrados positivos na região rural 102/147 (53,4\%) Triatoma longipennis, 35/43 (81,3\%) Triatoma barberi e 22/87 (25,2\%) ninfas; na região urbana $22 / 35(62,8 \%)$ Triatoma longipennis, 6/7 (85,7\%) Triatoma barberi e 2/11 (18,1\%) ninfas $s p$. Foi capturado apenas um exemplar de Triatoma dimidiata no município de La Barca, o qual se encontrava positivo para Trypanosoma cruzi (Figura 3).

Tabela 2 - Distribuição por espécie peri e intradomiciliar dos triatomíneos capturados

\begin{tabular}{lccc}
\hline & Urbano & \multicolumn{2}{c}{ Rural } \\
\cline { 3 - 4 } espécie/zona de captura & intradomicilio & intradomicílio & peridomicílio \\
\hline Triatoma longipennis & 34 & 49 & 98 \\
Triatoma barberi & 6 & 7 & 35 \\
Ninfas $s p$ & 11 & 12 & 75 \\
Triatoma dimidiata & 1 & & \\
\hline Total & 52 & 68 & 208 \\
\hline
\end{tabular}




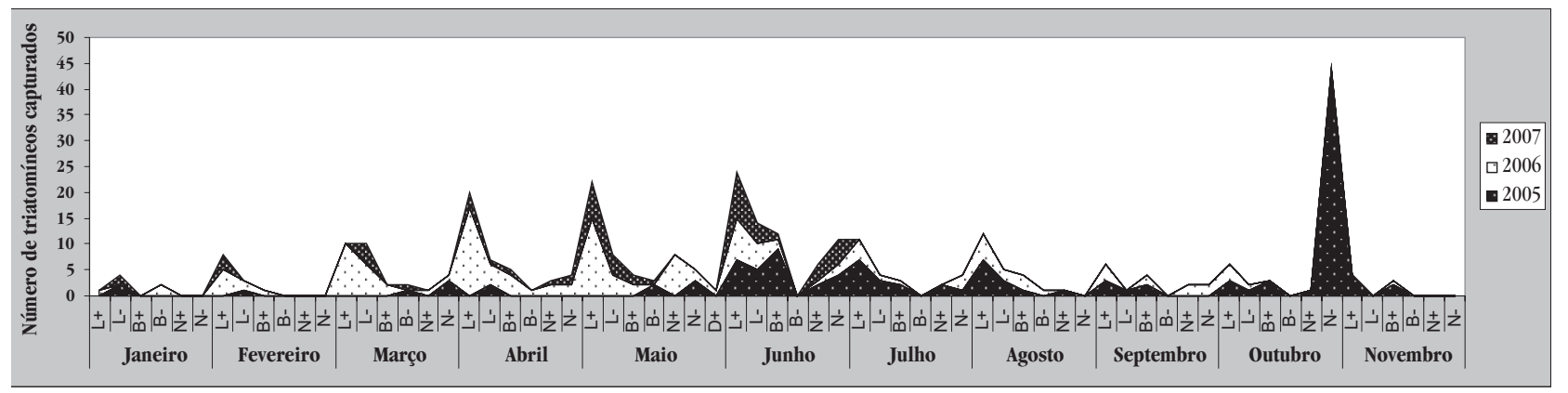

L+:Triatoma longipennis positivo, L-: Triatoma longipennis negativo, B+: Triatoma barberi positivo, B-: Triatoma barberi negativo, N+: ninfas sp positivas, N-: ninfas sp negativas, D+: Triatoma dimidiata positivo.

Figura 2 - Gráfico da sazonalidade das diferentes espécies de triatomíneos entre os anos 2005 a 2007.

Tabela 3 - Amostragem de triatomíneos capturados por mês em 13 localidades da região Ciénega do Estado de Jalisco, México no período 2005-2007.

\begin{tabular}{|c|c|c|c|c|c|c|c|c|c|c|c|c|c|c|c|c|c|c|c|c|c|c|}
\hline \multirow{2}{*}{$\begin{array}{c}\text { Ano } \\
\text { Mês/espécie }\end{array}$} & \multicolumn{7}{|c|}{2005} & \multicolumn{8}{|c|}{2006} & \multicolumn{7}{|c|}{2007} \\
\hline & L+ & L- & B+ & B- & $\mathrm{N}+$ & $\mathrm{N}-$ & Total & L+ & L- & $\mathbf{B +}$ & B- & $\mathrm{N}+$ & N- & D+ & Total & $\mathrm{L}+$ & L- & B+ & B- & $\mathbf{N +}$ & N- & Total \\
\hline Janeiro & & 2 & & & & & 2 & 1 & & & 2 & & & & 3 & & 2 & & & & & 2 \\
\hline Fevereiro & & 1 & & & & & 1 & 5 & 2 & 1 & & & & & 8 & 3 & & & & & & 3 \\
\hline Março & & & & & & 3 & 3 & 10 & 6 & 2 & & 1 & 1 & & 20 & & 4 & & 1 & & & 5 \\
\hline Abril & & 2 & & & & & 2 & 17 & 4 & 4 & 1 & 2 & 2 & & 30 & 3 & 1 & 1 & & 1 & 2 & 8 \\
\hline Maio & & & & 2 & & 3 & 5 & 15 & 4 & 2 & & 8 & 2 & 1 & 32 & 7 & 4 & 2 & 1 & & & 14 \\
\hline Junho & 7 & 5 & 9 & & 2 & 4 & 27 & 8 & 5 & 2 & & 1 & 2 & & 18 & 9 & 4 & 1 & & 3 & 5 & 22 \\
\hline Julho & 7 & 3 & 2 & & 2 & 1 & 15 & 4 & 1 & 1 & & & 3 & & 10 & & & & & & & \\
\hline Agosto & 6 & 3 & 1 & & 1 & & 11 & 5 & 2 & 3 & 1 & & & & 10 & & & & & & & \\
\hline Setembro & 3 & 1 & 2 & & & & 6 & 3 & & 2 & & 2 & 2 & & 9 & & & & & & & \\
\hline Outubro & 3 & 1 & 2 & & 1 & 41 & 48 & 3 & 1 & & & & 3 & & 7 & & & & & & & \\
\hline Novembro & 4 & & 2 & & & & 6 & & & 1 & & & & & 1 & & & & & & & \\
\hline Total & 30 & 18 & 18 & 2 & 6 & 52 & 126 & 71 & 25 & 18 & 4 & 14 & 15 & 1 & 148 & 22 & 15 & 4 & 2 & 4 & 7 & 54 \\
\hline
\end{tabular}

L+: Triatoma longipennis positivo, L-: Triatoma longipennis negativo, B+: Triatoma barberi positivo, B-: Triatoma barberi negativo, N+: ninfas positivas, N-: ninfas negativas, D+: Triatoma dimidiata positivo.

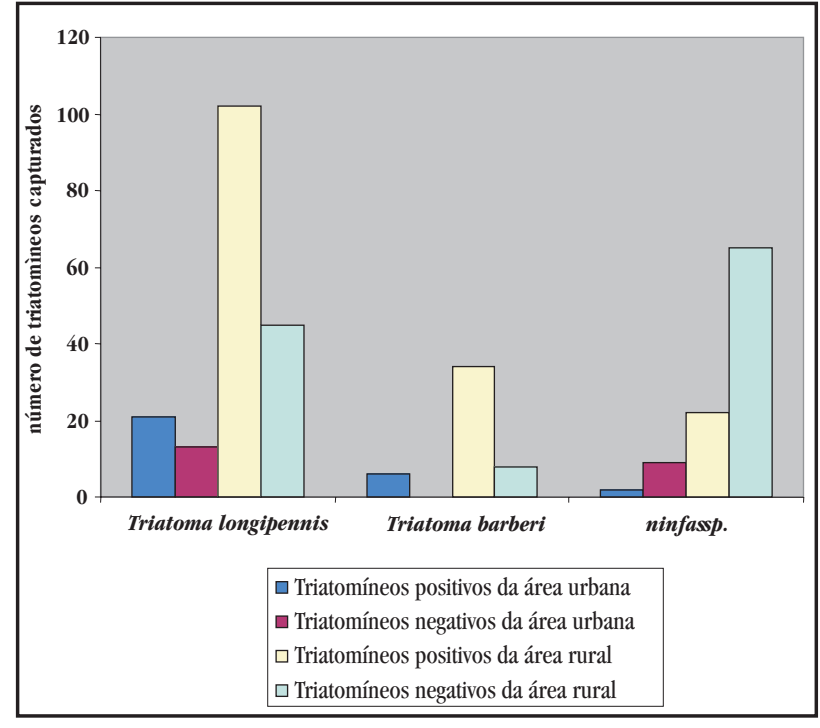

Figura 3 - Níveis de infecção de diferentes espécies de triatomíneos por Trypanosoma cruzi em área rural e urbana da região Ciénega $(p<0,05)$.

\section{DISCUSSÃo}

O sucesso do controle vetorial na América do Sul diminuiu o número de pessoas infetadas de 16-18 milhões (1991) para 10-12 milhões (1999). A transmissão da doença foi controlada em alguns países como Uruguai, Chile e em alguns estados brasileiros e quatro províncias argentinas ${ }^{18} 23$. No entanto, em países da América Central e México cerca de 2,3 milhões de pessoas se encontram infetadas, com uma incidência anual de 70 mil indivíduos ${ }^{24}$.

0 México não possui um programa efetivo em relação aos aspectos de diagnóstico, vigilância, prevenção e controle da doença, havendo poucos trabalhos publicados sobre o mesmo ${ }^{67}$.

A realização de diversos estudos na região oeste do Estado de Jalisco, México, tem mostrado que a invasão de triatomíneos de hábitos peridomiciliar na área domiciliar, ocorre na época de seca, antecedendo ao período chuvoso. Este fator representa um elevado risco de transmissão de Trypanosoma cruzi, o que pode acarretar um aumento da infecção nas populações urbanas e rurais que estão dentro da área de distribuiçãa $0^{2} 151617$.

Em nosso trabalho observamos que á época de maiores índices de captura ocorriam no período anterior as chuvas. Estudos têm demonstrado que a temperatura é o principal fator para 0 desenvolvimento acelerado do ciclo biológico dos triatomíneos. Temperaturas baixas influenciam o processo de embriogênese e o desenvolvimento das mudas, retardando o seu ciclo evolutivo. Já as temperaturas elevadas ocasionam desidratação e elevada mortalidade, diminuindo a densidade dos mesmos. As mudanças climáticas obrigam os triatomíneos (silvestres e peridomésticos) a procurarem um novo abrigo, tornando a área domiciliar 
favorável para sua reprodução e aumentando, portanto, o risco de transmissão do parasita ${ }^{4}$.

Ao invadir os domicílios, os triatomíneos entram em contato com lugares propícios para sobrevivência e reprodução. As casas são geralmente construídas com barro, lâminas (asbesto, zinco, entre outras) e pau-a-pique, tornando o ambiente favorável para o encontro destes vetores ${ }^{424}$. Estes fatores justificam 0 alto $(84,1 \%)$ índice de captura nos domicílios rurais, sendo que destes, $84,5 \%$ foram positivos para Trypanosoma cruzi (Figura 3). Estes dados podem justificar os achados de Yerenas e cols que detectaram uma soroprevalência de $21,6 \%$ na população rural do município de La Barca, Jalisco ${ }^{25}$.

0 indicador de colonização por espécie não foi calculado porque algumas casas estavam infestadas com mais de uma espécie de triatomíneo e as ninfas são muito similares, não existindo chaves de classificação para diferenciá-las a nível espécie. Este mesmo fato ocorreu no trabalho realizado por Magallón-Gastélum e cols ${ }^{15}$, no ano de 2006. Em nosso estudo observou-se um comportamento contrário na positividade das ninfas $s p$ quando comparado com as formas adultas (Triatoma longipennis e Triatoma barberi), sugerindo uma menor suscetibilidade ao parasito nos estádios jovens.

Os índices de infestação por triatomíneos em áreas intradomiciliar e peridomiciliar descritos neste trabalho são similares aos índices encontrados por outros estudos realizados em outras regiões do Estado de Jalisco ${ }^{22}$. Os indicadores de infestação (a maioria no ambiente peridomiciliar) podem ser justificados, provavelmente, pelo comportamento ornitofílico das espécies encontradas em nosso estudo, como mencionado por outros trabalhos ${ }^{15} 16$.

Triatoma dimidiata é o segundo vetor de maior importância na doença de Chagas nos países andinos (Colômbia, Equador e Venezuela), nos países da América Central (Nicarágua, Guatemala, Honduras, Costa Rica e Belize) e no México. Esta espécie se caracteriza pela sua capacidade de reinfestação das casas após tratamento com inseticidas. Os estudos de mobilidade indicam que tanto as ninfas como os adultos apresentam capacidade de deslocamento, influenciando diretamente nas estratégias de controle ${ }^{8111^{22}}$. No México, o Triatoma dimidiata pode ser encontrado na península de Yucatán (Yucatán, Quintana Rôo), istmo de Tehuantepec, e ao longo do litoral do Golfo do México (Veracruz, Tabasco Campeche,) e do Oceano Pacífico (Chiapas, Oaxaca, Guerrero). Dumonteill \& Gourbière demonstraram que, no estado de Yucatán, o Triatoma dimidiata era mais abundante nos domicílios nos meses de abril a junho (correspondentes á época de calor), apresentando maiores índices de infecção por Trypanosoma cruzi e sugerindo um maior risco de transmissão neste período $0^{9}$. Este triatomíneo é considerado um dos melhores transmissores do Trypanosoma cruzi apesar da baixa densidade populacional ${ }^{67811121623}$, e de ser considerada como uma das espécies de maior adaptabilidade aos diferentes habitats ${ }^{121626}$. Não se tem registros de Triatoma dimidiata em regiões montanhosas do centro do país. Em nosso trabalho foi encontrado apenas um exemplar de Triatoma dimidiata, o qual era positivo para Trypanosoma cruzi. Este é o primeiro registro desta espécie positivo na região Ciénega, sugerindo que este vetor esteja se adaptando a novos ecótopos, tornando-se um candidato para transmissão do parasita.

Martinez-Ibarra e cols ${ }^{17}$ consideram o Triatoma longipennis como principal transmissor do Trypanosoma cruzi por ser encontrado em grandes quantidades e apresentar índices de infecção natural mais alto quando comparado a outras espécies ${ }^{16}$. Nosso estudo demonstra que esta espécie foi a mais abundante, porém, o Triatoma barberi apresentou maior índice de infecção natural.

Em resumo, nosso trabalho mostra que na região Ciénega, Estado de Jalisco, o índice de triatomíneos positivos para Trypanosoma cruzi é elevado (57,3\%) quando comparado com outras regiões do mesmo estado, sendo a maior frequiência nos meses de abril, maio e junho correspondendo à época da seca. Demonstramos que as duas espécies mais importantes são Triatoma longipennise Triatoma barberi sendo a primeira mais abundante e a segunda caracterizada por apresentar maior índice de positividade. Além disso, registrou-se o primeiro achado de Triatoma dimidiata positivo nesta região. Os altos índices de infecção dos vetores capturados sugerem uma elevada exposição das pessoas ao Trypanosoma cruzi.

\section{REFERÊNCIAS}

1. Acha PN, Szyfres B. Zoonosis y enfermedades transmisibles comunes al hombre y a los animales. Organización Panamericana de la Salud Organización Mundial de la Salud, Publicación científica nº 503, 1986.

2. Brenière SF, Bosseno M, Magallón-Gastelúm E, Ruvalcaba EGC, Gutierrez MS, Luna ECM, Basulto JT, Mathieu-Daudé F, Walter A, Lozano-Kasten F. Peridomestic colonization of Triatoma longipennis (Hemiptera, Reduviidae) and Triatoma barberi (Hemiptera, Reduviidae) in a rural community with active transmission of Trypanosoma cruzi in Jalisco State, Mexico. Acta Tropica 101: 249-257, 2007.

3. Brener Z, Andrade ZA. 0 parásito. In: Brener Z, Andrade ZA (eds) Relações hospedeiro parásito en Trypanosoma cruzi e Doença de Chagas. Editora Guanabara Koogan, Rio de Janeiro, p. 530, 1979.

4. Cabello DR, Lizano E, Valderrama A. Efecto de la temperatura y la dieta sobre el ciclo de vida de Rhodnius neivai LENT, 1953 (Hemiptera: Reduviidae). Caribbean Journal of Science 34: 99-105, 1998.

5. Contreras FT, Yerenas MV, Gutierrez MS, Anaya MR, Corder AJ. Serological followup of Trypanosoma cruzi infection from 1987 to 1994 in individuals studies in 50 counties of the State of Jalisco, Mexico. Revista da Sociedade Brasileira de Medicina Tropical 33: 591-596, 2000.

6. Cruz-Reyes A, Pickering-López JM. Base de datos “CHAGMEX® 1928-2004, sobre la Enfermedad de Chagas en México, con un enfoque biológico, geográfico y socioeconómico. http://www.ibiologia.unam.mx (Acesso: 29/10/2007), 2005.

7. Cruz-Reyes A, Pickering-López JM. Chagas disease in Mexico: an analysis of geographical distribution during the past 76 years. Memórias do Instituto Oswaldo Cruz 101: 345-354, 2006.

8. Dorn PL, Monroy C, Curtis A. Triatoma dimidiata (Latreille, 1811): A review of its diversity across its geographic range and the relationship among populations. Infection, Genetics and Evolution 7: 343-352, 2007.

9. Dumonteil, E, Gourbière $S$. Predicción de la abundancia y tasa de infección de Triatoma dimidiata: un mapa de riesgo de transmisión natural de la enfermedad de Chagas en la Península de Yucatán, México. Revista Biomédica. 15: 221-231, 2004.

10. Galvão C, Carcavallo RU, Rocha DS, Jurberg J. A checklist of the current valid species of the subfamily Triatominae jeannel, 1919 (Hemiptera, Reduviidae) 
and their geographical distribution, with nomenclatural and taxonomic notes. Zootaxa 202: 1-36, 2003.

11. Guzmán-Bracho MC. Epidemiology of Chagas disease in Mexico: an update 2001. Trends in Parasilotogy 17: 372-376, 2002.

12. Guzmán-Marín ES, Barrera-Pérez MA, Rodríguez-Félix ME; Zavala-Velázquez JE. Hábitos biológicos de Triatoma dimidiata en el estado de Yucatán, México. Revista Biomédica 3: 125-131, 1992.

13. Jiménez ML, Palacios C. Estudio sobre la incidencia de la chinche piedrera Dipetalogaster maximus (Uhler) (Hemiptera: Heteroptera: Reduviidae) vector de Trypanosoma cruzi en zonas urbanas de La Paz, BCS, México. Annais del Instituto de Biología de la Universidad Nacional Autonoma de México 70: 215221, 1999 .

14. Lent H, Wigodzinsky P. Revision of the triatominae (Hemiptera: Reduviidae), and their significance as vectors of Chagas disease. Bulletin of the American Museum of Natural History 163: 520, 1979.

15. Magallón-Gastélum E, Lozano-Kasten F, Gutierrez MS, Flores-Perez A, Sanchez B, Espinoza B, Bosseno M, Brenière SF. Epidemiological risk for Trypanosoma cruzi transmission by species of Phyllosoma complex in the occidental part of Mexico. Acta Tropica 97: 331-338, 2006.

16. Magallón-Gastélum E, Magdaleno-Peñaloza NC, Kattahain-Duchateau G, Trujillo-Contreras F, Lozano-Kasten FJ, Hernández-Gutiérrez RJ. Distribución de los vectores de la enfermedad de Chagas Hemiptera: Reduviidae: Triatominae en el estado de Jalisco, México. Revista Biomédica 9: 151-157, 1998.

17. Martinez-Ibarra JA, Barcenas-Ortega NM, Nogueda-Torres B, Alejandre-Aguilar R, Lino-Rodríguez M, Magallón-Gastélum E, Lopez-Martinez V, Romero-Napoles J. Role of two Triatoma Hemiptera: Reduviidae: Triatominae species in the transmission of Trypanosoma cruzi Kinetoplastida: Trypanosomatidae to man in the west coast of Mexico. Memórias do Instituto Oswaldo Cruz 96: 141-144, 2001.
18. Salazar-Shetino PM, Haro-Arteaga I, Ubribarren-Berrueta T. Chagas disease in México. Parasitology Today 4: 348-352, 1988.

19. Schofield CJ, Dias JCP. The Southern Cone programme against Chagas' disease. Advances in Parasitology 42:1-25,1999.

20. Secretaría de Salud Jalisco. Taller Nacional de Vigilancia Epidemiológica. Prevención y control de la enfermedad de Chagas en México. Organización Panamericana de la Salud. http://www.paho.org/spanish/AD/DPC/CD/chagas (Acesso: 29/10/2007), 2003

21. Velasco-Castrejón 0, Guzmán-Bracho C. Importancia de la Enfermedad de Chagas en México. Revista Latino-americana de Microbiologia 28: 275-283, 1986.

22. Vidal-Acosta V, Ibáñez-Bernal S, Martínez-Campos C. Infección natural de chinches Triatominae con Trypanosoma cruzi asociadas a la vivienda humana en México. Salud Pública de México 42: 496-503, 2000.

23. Walter A, Lozano-Kasten F, Bosseno M, Ruvalcaba EGC, Gutierrez MS, Luna CEM, Baunaure F, Phélinas P, Magallón-Gastélum E, Brenière SF. Peridomicilary habitat and risk factors for Triatoma Infestation in a rural community of the Mexican occident. The American Journal Tropical Medicine and Hygiene 76: 508-515, 2007.

24. World Health Organization. Control of Chagas disease. Technical Report Series 905: 40-55, 2002.

25. Yerenas MLA, Contreras FT, Hernandez CG, Hernandez LAG, Zarate AC, Anguiano RRL, Flores PT, Lara JPR, Sanchez AUF, Aceves FFC, Gonzalez AF, Briceño PC. Estudio seroepidemiologico de la enfermedad en la zona rural del municipio de La barca, Jalisco, Mexico 2005. In: Resumos do XLII Congresso da Sociedade Brasileira de Medicina Tropical, Brasilia p. 197, 2006.

26. Zarate LG, Zarate RA. Cheklist of the triatominae (Hemiptera: reduviidae) of Mexico. International Journal Entomology 27: 102-127, 1985.

27. Zeledon R. Vectores de la Enfermedad de Chagas y sus características ecofisiológicas. Interciencia 8: 384-395, 1983. 